\title{
INVESTIGATIONS IN HARMONIC ANALYSIS $\left({ }^{1}\right)$
}

\author{
BY \\ H. J. REITER
}

This paper is concerned with the theory of ideals in the algebra $L^{1}$ of integrable functions on a locally compact abelian group.

After some preliminaries an analytical proof is given of the known theorem that an analytic function of a Fourier transform represents again a Fourier transform (p. 406). Then, in part I, the continuous homomorphisms of closed ideals $I$ of $L^{1}$ upon $C$, the field of complex numbers, are studied. Any such homomorphism is given by a Fourier transform and, if $I_{0}$ is its kernel, the quotient-algebra $I / I_{0}$, normed in the usual way, is not only algebraically isomorphic, but also isometric with $C$ (Theorem 1.2). Another result states that homomorphic groups have homomorphic $L^{1}$-algebras and that a corresponding property of isometry holds (Theorem 1.3).

In part II, which may be read independently of part I, a theorem of S. Mandelbrojt and S. Agmon, which generalizes Wiener's theorem on the translates of a function in $L^{1}$, is extended to groups (Theorem 2.2). Several generalizations of Wiener's classical theorem have been published in the past few years; references to the literature are given on p. 422 . The rest of part II is devoted to some applications (pp. 422-425).

In conclusion it should be said that the work is carried out in abstract generality, with the methods, and in the spirit, of analysis, which is then applied to algebra.

To Professors S. Mandelbrojt, B. L. van der Waerden, and A. Weil I owe my mathematical education. The inspiration which I have received in their lectures, in letters, and above all in personal contact, is at the base of this work; may I here express my gratitude.

\section{BASIC CONCEPTS AND Results. Notation}

Groups and Fourier transforms. The standard work of reference is A. Weil's book [14]; cf. also [4].

For the definition of a topological group see $[14$, p. 9]. The additive group of real numbers (the "real axis") is denoted by $R^{1}, p$-dimensional space by $R^{p}$. The group operation will always be denoted multiplicatively (it is hoped that this will not cause confusion in the case of $R^{1}$ ). The identity (unit element) of the group is denoted by $\mathbf{e}$.

We shall deal only with locally compact abelian groups $G$. By $G$ we denote

Presented to the Society, December 28, 1951; received by the editors January 12, 1952.

(1) This paper was submitted to the Faculty of the Rice Institute as a doctoral dissertation. The author wishes to express his thanks for the generous award of a fellowship. 
the dual group, by $(x, x)$ the value for $x \in G$ of the character defined by $x \in G$. For $R^{1}$ the symbol $(\mathrm{x}, x)$ may be regarded simply as an abbreviation of $\exp (2 \pi i x x)$ and $R^{1}$ is the same as $R^{1}$.

The measure of a set $\mathbf{S} \subset \mathbf{G}(S \subset G)$ is denoted by $m(S)(m(S)) . L^{1}, L^{2}, L^{\infty}$ denote, respectively, the space of complex-valued functions on $G$ which are integrable, of integrable square, measurable, and essentially bounded. The last notation, a slight departure from Weil's, is used in [4]. Functions in $L^{1}, L^{2}, L^{\infty}$ will be denoted, respectively, by $f(x), \cdots, F(x), \cdots, \phi(x), \cdots$. We use a dot to indicate the conjugate of a complex number and we write $f^{*}(x), \phi^{*}(x)$ for $f^{*}\left(x^{-1}\right), \phi^{*}\left(x^{-1}\right)$.

The Faltung of two functions in $L^{1}$ is

$$
f * \xi=f * g(x)=\int f(y) \xi\left(x y^{-1}\right) d y .
$$

We shall also use the Faltung $\boldsymbol{f} * \boldsymbol{\phi}(\boldsymbol{x}), \boldsymbol{\phi} \in L^{\infty}$. Sometimes we shall write $\boldsymbol{f}(\mathrm{x}) * \boldsymbol{\phi}$ or even $\boldsymbol{f}(\mathrm{x}) * \boldsymbol{\phi}(\mathrm{x})$, according to convenience. If $\phi_{1}=\boldsymbol{f} * \boldsymbol{\phi}$, then $\phi_{1}^{*}=f^{*} * \phi^{*}$.

The Fourier transform of $f \in L^{1}$ is $\left({ }^{2}\right)$

$$
f(x)=\int f(x)(x, x) \cdot d x .
$$

This is the definition adopted in $[4$, p. 87] rather than that used by Weil $\left[14\right.$, p. 112] - the difference is irrelevant. For $G=R^{1}$ it represents a slight, but convenient, deviation from the classical definition.

Banach spaces. For the definition and properties of Banach spaces $B$ over the field of complex numbers, and the notation, we refer to Hille's book [8].

$L^{1}$ and $L^{\infty}$ are Banach spaces, the norms being, respectively,

$$
\|f\|_{1}=\int|f(x)| d x, \quad\|\phi\|_{\infty}=\text { ess. } \sup |\phi(x)| \quad(x \in G) .
$$

The dual space of $L^{1}$ is $L^{\infty}$; we shall write the general bounded linear functional on $L^{1}$ in the form

$$
\int f(x) \varphi(x) d x
$$

The following "distance theorem" is a reformulation of a well known result [8, Theorem 2.9.4]:

Let $L$ be a closed linear subspace of $B$ and define for $x_{1} \in B$

(2) Thus a function in $L^{1}$ is indicated by a letter in boldface type and its Fourier transform by the same letter in italic type. This notation, considerably different from that used by French authors, was dictated by technical necessities of printing. Likewise for the use of the dot instead of the customary bar. 


$$
\operatorname{dist}\left\{x_{1}, L\right\}=\inf \left\|x_{1}-x_{0}\right\| \quad\left(x_{0} \in L\right) .
$$

If there is no bounded linear functional $x^{*}(x)$ satisfying

$$
\begin{aligned}
& x^{*}\left(x_{1}\right)=1, \\
& x^{*}\left(x_{0}\right)=0
\end{aligned} \quad \text { for all } x_{0} \in L,
$$

then $x_{1} \in L$

If there are bounded linear functionals satisfying condition $(*)$, then

$$
\text { dist }\left\{x_{1}, L\right\}=1 / \min \left\|x^{*}\right\| \text {, }
$$

where $x^{*}$ ranges over all these functionals.

This is an immediate consequence of Theorem 2.9.4 in [8] and the fact that if $x^{*}$ satisfies $\left(^{*}\right)$ then $x^{*}\left(x_{1}-x_{0}\right)=1$, and hence $\left\|x^{*}\right\| \geqq 1 /\left\|x_{1}-x_{0}\right\|$.

This theorem will be applied, in the space $L^{1}$, to calculate the distance in some cases (cf. [3, chap. III]).

The function $\phi(x)$ is called orthogonal to $f(x): \phi \perp f$, if $\int f(x) \phi^{\bullet}(x) d x=0$. It is called orthogonal to the linear subspace $I \subset L^{1}: \phi \perp I$, if $\phi \perp f$ for all $f \in I$. If $\phi \perp L^{1}$, then $\phi$ vanishes almost everywhere.

The $L^{1}$-algebra. $L^{1}$ is not only a Banach space, but also a commutative Banach algebra [8], multiplication being defined by the Faltung.

A (closed) ideal in the algebra $L^{1}$ is defined as a (closed) linear subspace of $L^{1}$ which contains, with a function $f$, all products $f * \xi$, for any $\xi \in L^{1}$. Closed ideals will be denoted by $I$.

A closed linear subspace is an ideal if and only if it is "invariant under translation," i.e., contains, with a function $f(x)$, all "translates" $f\left(x y^{-1}\right)$, for any (fixed) $y \in G$. For the sufficiency see [6, p. 127]. The necessity is proved here, for the sake of completeness.

Consider any $f \in I$ and any $\boldsymbol{g} \in L^{1}$. Then, by hypothesis, $f * \boldsymbol{g} \in I$ and hence if $\int \boldsymbol{f}(\mathbf{x}) \boldsymbol{\phi}^{\bullet}(\mathbf{x}) d \mathbf{x}=0$ for all $\boldsymbol{f} \in I$, then

$$
\int \boldsymbol{\phi}^{\bullet}(\mathbf{x}) d \mathbf{x} \int \boldsymbol{f}\left(\mathbf{x} \mathbf{y}^{-1}\right) \boldsymbol{g}(\mathbf{y}) d y=0
$$

or

$$
\int \boldsymbol{g}(\mathrm{y}) d \boldsymbol{y} \int \boldsymbol{f}\left(\mathbf{x} \mathbf{y}^{-1}\right) \boldsymbol{\phi}^{\bullet}(\mathbf{x}) d \mathbf{x}=0 .
$$

Since $\boldsymbol{g}(\boldsymbol{y})$ is arbitrary, it follows that

$$
\int \boldsymbol{f}\left(\mathrm{x} \mathrm{y}^{-1}\right) \boldsymbol{\phi}^{\bullet}(\mathrm{x}) d \mathrm{x}=0 .
$$

Since this holds for any $\phi \perp I$, it follows that $f\left(x y^{-1}\right) \in I$, for any (fixed) $y \in G$. The intersection of the zeros of the Fourier transforms of all functions in 
$I$, which is a closed set in $G$, is called the co-spectrum of $I$ by L. Schwartz [12]. We shall denote it by $Z_{I}$.

By $I(x, x)^{\bullet}$ we shall mean the "rotated" ideal obtained by replacing all $\boldsymbol{f}(\mathbf{x}) \in \boldsymbol{I}$ by $\boldsymbol{f}(\mathbf{x})(\mathbf{x}, x)^{\bullet}, x$ being any (fixed) element of $G$.

\section{PreliminaRY CONSIDERATIONS}

Let $S$ be a compact, symmetrical neighborhood of the identity $e \in G$ and denote by $S(x)$ the characteristic function of $S$ :

$$
\begin{aligned}
& S(x)=1, \\
& S(x)=0,
\end{aligned}
$$$$
x \in S \text {, }
$$$$
x \in \mathcal{C} \text {. }
$$

This will be used as a standard notation throughout, and neighborhoods $S, S^{\prime}, S_{n}$, etc., will always be supposed to have the above properties (without explicit mention).

Define now

$$
\mathbf{S}(\mathbf{x})=\int S(x)(\mathbf{x}, x) d x .
$$

Since $S^{-1}=S, S(x)$ is real and $S\left(x^{-1}\right)=S(x)$. If $G=R^{1}$ and $S=[-\epsilon / 2, \epsilon / 2]$, $S(x)=(\sin \pi \in x) / \pi x ;$ if $G$ is compact and $S=\{e\}$, then $S(x)=1$.

Consider now the Faltung

$$
S * S(x)=m(x S \cap S) .
$$

By the theorem of Plancherel-Weil

$$
S * S(x)=\int S(\mathbf{x})^{2}(\mathbf{x}, x) \cdot d \mathbf{x} .
$$

In the applications we shall "normalize" the above Faltung by means of the factor $1 / m(S)$. The function thus obtained takes values between 0 and 1 ; it is 1 for $x=e$ and vanishes outside the neighborhood $S^{2}$.

The function $S(x)^{2}$ is non-negative and has the following properties $\left({ }^{3}\right)$ :

$$
m^{-1}(S) \int S(x)^{2} d x=m^{-1}(S)\left\|S(x)^{2}\right\|_{1}=\left(m^{-1 / 2}(S)\|S(x)\|_{2}\right)^{2}=1 .
$$

This follows immediately from the theorem of Plancherel-Weil, applied to $S(x)$ and $S(x)$.

Given $\epsilon>0$, for any (fixed) $y \in G$

$$
m^{-1}(S)\left\|S\left(y^{-1} x\right)^{2}-S(x)^{2}\right\|_{1}<\epsilon,
$$

(8) We shall denote the norm in $L^{2}$ (and in the corresponding space $L^{2}$ ) by $\|F\|_{2}$ $=\left\{\int|F(x)|^{2} d x\right\}^{1 / 2}$. 
provided $S$ is small ( $S \subset U$, a neighborhood of $e$ depending on $\epsilon$ and $y$ ).

By Schwarz's inequality

$$
\begin{aligned}
m^{-1}(S) \|\left\{S\left(y^{-1} \mathbf{x}\right)+\boldsymbol{S}(\mathbf{x})\right\} & \left\{\boldsymbol{S}\left(\boldsymbol{y}^{-1} \mathbf{x}\right)-\boldsymbol{S}(\mathbf{x})\right\} \|_{1} \\
& \leqq m^{-1}(S)\left\|\boldsymbol{S}\left(\boldsymbol{y}^{-1} \mathbf{x}\right)+\boldsymbol{S}(\mathbf{x})\right\|_{2}\left\|\boldsymbol{S}\left(\boldsymbol{y}^{-1} \mathbf{x}\right)-\boldsymbol{S}(\mathbf{x})\right\|_{2} .
\end{aligned}
$$

By the theorem of Plancherel-Weil this is equal to

$$
\begin{aligned}
& m^{-1 / 2}(S)\left\|S(x)\left\{(y, x)^{\bullet}+1\right\}\right\|_{2} m^{-1 / 2}(S)\left\|S(x)\left\{(y, x)^{\bullet}-1\right\}\right\|_{2} \\
& \leqq 2\left(m^{-1}(S) \int_{S}\left|(y, x)^{\bullet}-1\right|^{2} d x\right)^{1 / 2}<\epsilon,
\end{aligned}
$$

provided $S \subset U$, where the neighborhood $U$ of $e$ is such that $|(y, x)-1|$ $<\epsilon / 2$ for all $x \in U$ and the given $y$.

Take now two neighborhoods $S$ and $S^{\prime}$ (cf. the convention concerning the notation on p. 404). Then the product $S S^{\prime}$ is a neighborhood of the same kind.

Consider the characteristic functions

$$
S(x) \text { and } S S^{\prime}(x)
$$

(the use of two letters to denote a function is perhaps somewhat unusual, but convenient here). The normalized Faltung of these functions,

$$
m^{-1}(S) S * S S^{\prime}(x)=m^{-1}(S) m\left(x S \cap S S^{\prime}\right),
$$

takes values between 0 and 1 ; it is 1 for $x \in S^{\prime}$, vanishes outside $S^{2} S^{\prime}$, and is the Fourier transform of

$$
m^{-1}(S) S(x) \cdot S S^{\prime}(x)
$$

We shall need later the following estimate:

$$
\left\|S(x) S S^{\prime}(x)-S(x)^{2}\right\|_{1} \leqq\left\{m\left(S S^{\prime}\right)-m(S)\right\}^{1 / 2} m^{1 / 2}(S) .
$$

Indeed,

$$
\begin{aligned}
& \left\|\boldsymbol{S}(\mathbf{x})\left\{\mathbf{S} \mathbf{S}^{\prime}(\mathbf{x})-\mathbf{S}(\mathbf{x})\right\}\right\|_{1} \leqq\|\boldsymbol{S}(\mathbf{x})\|_{2}\left\|\mathbf{S} \boldsymbol{S}^{\prime}(\mathbf{x})-\mathbf{S}(\mathbf{x})\right\|_{2}= \\
& \|S(x)\|_{2}\left\|S S^{\prime}(x)-S(x)\right\|_{2}=m^{1 / 2}(S)\left\{m\left(S S^{\prime}\right)-m(S)\right\}^{1 / 2}
\end{aligned}
$$

We shall also need a stronger form of property (ii): Given any (fixed) compact set $C \subset G$, then for any $\epsilon>0$

$$
m^{-1}(S)\left\|S\left(y^{-1} x\right)^{2}-S(x)^{2}\right\|_{1}<\epsilon
$$

for all $y \in C$, provided $S$ is small ( $S \subset U$, a neighborhood of $e$ which depends on $\boldsymbol{\epsilon}$ and $\boldsymbol{C}$ ).

Referring to the proof of (ii), it is required to show that there exists a neighborhood $U$ of $e$ such that $|(y, x)-1|<\epsilon / 2$ for all $y \in C$ and all $x \in U$ (this is obvious in the case $G=R^{1}$; in the general case it is precisely the defini- 
tion of the topology of the dual group given by Pontrjagin).

The character $(y, x)$ is a continuous function on $G \times G[14$, p. 101]. Thus, given $\epsilon>0$, for each point $y_{\imath} \in C$ there are neighborhoods $V_{\imath}$ of $y_{\iota}$ and $U_{\imath}$ of $e$ such that $|(y, x)-1|=\left|(y, x)-\left(y_{\imath}, e\right)\right|<\epsilon / 2$ for all $y \in V_{\imath}$ and all $x \in U_{\imath}$.

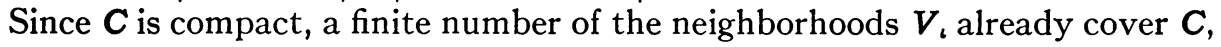
and we may take for $U$ any neighborhood of $e$ contained in the intersection of the corresponding neighborhoods $U_{l}$. Thus (ii') is proved $\left({ }^{4}\right)$.

Another estimate to be applied later is the following:

Let $C \subset G$ be a compact set and let $\epsilon>0$ be given. Then

$$
m^{-1}(S)\left\|\boldsymbol{S}\left(\boldsymbol{y}^{-1} \mathbf{x}\right) \mathbf{S} \boldsymbol{S}^{\prime}\left(\boldsymbol{y}^{-1} \mathbf{x}\right)-\boldsymbol{S}(\mathbf{x}) \boldsymbol{S} \boldsymbol{S}^{\prime}(\mathbf{x})\right\|_{1}<\boldsymbol{\epsilon}\left(m\left(S S^{\prime}\right) / m(S)\right)^{1 / 2}
$$

for all $y \in C$, provided both $S$ and $S^{\prime}$ are chosen small (namely, such that $S S^{\prime} \subset U$, where $U$ is some neighborhood of $e$ depending on $\epsilon$ and $C$ ).

The proof is analogous to that of properties (ii) and (ii'), $U$ being such that $|(y, x)-1|<\epsilon / 2$ for all $y \in C, x \in U$.

We also have

$$
m^{-1}(S)\left\|\boldsymbol{S}(\mathbf{x}) \boldsymbol{S} \boldsymbol{S}^{\prime}(\mathbf{x})\right\|_{1} \leqq\left(m\left(S S^{\prime}\right) / m(S)\right)^{1 / 2} .
$$

\section{ANALytic FUnCtions OF Fourier transforms}

THEOREM. Let $\boldsymbol{f}(\mathbf{x})$ be a function in $L^{1}$ and let $C \subset G$ be a compact set. Denote by $\Gamma$ the set of points $z$, in the complex plane, of the form $z=f(x), x \in C$. Let now $A(z)$ be a (single-valued) analytic function, regular at all points of $\Gamma$. Then there is a function in $L^{1}$ whose Fourier transform is equal to $A(f(x))$ for all $x \in C$.

This theorem is proved in two steps: first it is proved locally, i.e., for a sufficiently small neighborhood of any (fixed) point $x_{0} \in C$; then the extension to the whole set $C$ is carried out. The proof is modelled after that given by Carleman for the case $G=R^{1}[3$, chap. IV $]\left({ }^{5}\right)$.

Let $x_{0}$ be any (fixed) point of $C$ and expand $A(z)$ in a power series about $z_{0}=f\left(x_{0}\right)$. We may write, for $x$ in some (small) neighborhood of $x_{0}$.

$$
A(f(x))=A\left(f\left(x_{0}\right)\right)+\sum_{n=1}^{\infty} c_{n}\left(f(x)-f\left(x_{0}\right)\right)^{n} .
$$

Consider now the Fourier transform (cf. p. 405)

$$
s(x)=m^{-1}(S) S(x) * S S^{\prime}(x) .
$$

If both $S$ and $S^{\prime}$ are small, the function

$$
s\left(x_{0}^{-1} x\right) A\left(f\left(x_{0}\right)\right)+\sum_{n=1}^{\infty} c_{n}\left\{s\left(x_{0}^{-1} x\right)\left(f(x)-f\left(x_{0}\right)\right)\right\}^{n}
$$

(4) The reader may now turn immediately to part I (p. 409) or part II (p. 417).

(5) Professor A. Weil has directed my attention to Segal's paper [13]. Theorem 3.8 in [13] practically contains, as a special case, the theorem given here. 
exists for all $x \in G$ and coincides with $A(f(x))$ for $x \in x_{0} S^{\prime}$.

The product

$$
s\left(x_{0}^{-1} x\right)\left(f(x)-f\left(x_{0}\right)\right)
$$

is the Fourier transform of

$$
q(\mathbf{x})=\left[\mathbf{s}(\mathbf{x})\left(\mathbf{x}, x_{0}\right)\right] * \mathbf{f}(\mathbf{x})-\mathbf{s}(\mathbf{x})\left(\mathbf{x}, x_{0}\right) f\left(x_{0}\right)
$$

where

$$
s(\mathbf{x})=m^{-1}(S) S(x) S S^{\prime}(x) .
$$

Write now

$$
\begin{aligned}
q(x) & =\int s\left(x y^{-1}\right)\left(x y^{-1}, x_{0}\right) f(y) d y-s(x)\left(x, x_{0}\right) \int f(y)\left(y, x_{0}\right) \cdot d y \\
& =\int\left(x y^{-1}, x_{0}\right)\left\{s\left(x y^{-1}\right)-s(x)\right\} f(y) d y .
\end{aligned}
$$

Hence

$$
\|q(x)\|_{1} \leqq \int|f(y)| d y \int\left|s\left(x y^{-1}\right)-s(x)\right| d x .
$$

Given $\epsilon>0$, let $K \subset G$ be a compact set such that

$$
\int_{\mathcal{C}_{K}}|f(y)| d y<\epsilon
$$

By property (iv), pp. 406-408, the neighborhoods $S, S^{\prime}$ may also be so chosen that

$$
\left\|s\left(y^{-1} x\right)-s(x)\right\|_{1}<\epsilon\left(m\left(S S^{\prime}\right) / m(S)\right)^{1 / 2}
$$

for all $y \in K$. Keeping $S$ fixed, we may take $S^{\prime}$ so small that $m\left(S S^{\prime}\right)<2 m(S)\left({ }^{6}\right)$.

Under these conditions

$$
\begin{aligned}
\|q(x)\|_{1} & \leqq 2^{1 / 2} \epsilon \int_{K}|f(y)| d y+2\|s(x)\|_{1 \epsilon} \\
& \leqq \epsilon\left\{2^{1 / 2}\|f\|_{1}+2(2)^{1 / 2}\right\}
\end{aligned}
$$

Define now

$$
q_{n}(x)=q * q_{n-1}(x), \quad n>1, \quad q_{1}(x)=q(x) .
$$

Then

(6) This follows from the regularity of Haar measure (cf. [7]) and the fact that for any open set $O$ containing $S$ there is an $S^{\prime}$ such that $S S^{\prime} \subset O$. 


$$
\left\|\boldsymbol{q}_{n}\right\|_{1} \leqq\|q\|_{1}^{n},
$$$$
n \geqq 1 \text {. }
$$

By a suitable choice of the neighborhoods involved, we can thus obtain a function $\boldsymbol{q}(\mathrm{x})$ such that $\|\boldsymbol{q}\|_{1}<R$, where $R$ is the radius of convergence of the power series $\sum_{n=1}^{\infty} c_{n} z^{n}$. Then the series

$$
\left(\mathbf{x}, x_{0}\right) \mathbf{s}(\mathbf{x}) A\left(f\left(x_{0}\right)\right)+\sum_{n=1}^{\infty} c_{n} \boldsymbol{q}_{n}(\mathbf{x})
$$

converges in $L^{1}$-norm and its Fourier transform coincides with $A(f(x))$ for all $x \in x_{0} S^{\prime}$.

The theorem has now been proved locally; to extend it to the set $C$ as a whole, we proceed as follows:

To every point $x_{\iota} \in C$ there corresponds a (small) neighborhood $x_{\iota} V_{\iota}, V_{\iota}$ being a neighborhood of $e$, such that there exists a function $f_{t} \in L^{1}$ whose Fourier transform $f_{\imath}(x)$ coincides with $A(f(x))$ for all $x \in x_{\imath} V_{\imath}^{3}$. The reason for considering first $x_{\imath} V_{\imath}$ and then $x_{\imath} V_{\imath}^{3}$ will appear shortly. Since $C$ is compact, a finite number of those neighborhoods, say $x_{1} V_{1}, x_{2} V_{2}, \cdots, x_{N} V_{N}$, already cover $C$ :

$$
C \subset \bigcup_{r=1}^{N} x_{r} V_{r}
$$

Take a neighborhood $S$ so small that $S \subset V_{r}(1 \leqq r \leqq N)$; then

$$
C S \subset \bigcup_{r=1}^{N} x_{r} V_{r}^{2}
$$

Define now a partition of the set $C S$ as follows:

$$
C S_{1}=C S \cap x_{1} V_{1}^{2}, \quad C S_{n}=C S \cap x_{n} V_{n}^{2} \cap \bigcup_{r=1}^{n-1} C S_{r} \quad(2 \leqq n \leqq N) .
$$

Thus

$$
C S_{r} \subset x_{r} V_{r}^{2}, \quad C S_{r} S \subset x_{r} V_{r}^{3} \quad(1 \leqq r \leqq N) .
$$

Consider the functions $f_{r}(\mathbf{x}) \in L^{1}(1 \leqq r \leqq N)$ whose Fourier transforms $f_{r}(x)$ coincide "locally" with $A(f(x))$, i.e., for $x \in x_{r} V_{r}^{3}$, respectively.

Let $C S_{r}(x)$ be the characteristic function of the set $C S_{r}(1 \leqq r \leqq N)$. Then the Faltung

$$
m^{-1}(S) S(x) * C S_{r}(x)
$$

is the Fourier transform of

$$
m^{-1}(S) \mathbf{S}(\mathbf{x}) \cdot \mathbf{C S}_{r}(\mathbf{x})
$$

(the notation being the usual), and vanishes outside $C S_{r} S$. Moreover the sum 


$$
\sum_{r=1}^{N} m^{-1}(S) S(x) * C S_{r}(x)
$$

is just

$$
m^{-1}(S) S(x) * C S(x)=m^{-1}(S) m(x S \cap C S)
$$

and hence is 1 for $x \in C$.

Now the Fourier transform

$$
\Sigma(x)=\sum_{r=1}^{N} f_{r}(x)\left[m^{-1}(S) S(x) * C S_{r}(x)\right]
$$

coincides with $A(f(x))$ for all $x \in C$.

Indeed, if $x \in C$, then in the product

$$
f_{r}(x)\left[m^{-1}(S) S(x) * C S_{r}(x)\right]
$$

either the Faltung in the bracket vanishes or else, if it does not, $f_{r}(x)$ coincides with $A(f(x))$, since $C S_{r} S \subset x_{r} V_{r}^{3}$.

Thus, for all $x \in C, \Sigma(x)=A(f(x)) \cdot 1$ and the theorem is proved.

The following particular case should be especially noted:

If $\boldsymbol{f}(\mathrm{x}) \in L^{1}$ is such that $f(x) \neq 0$ on the compact set $C \subset G$, then there is a function $\boldsymbol{g}(\mathrm{x}) \in L^{1}$ such that

$$
g(x)=1 / f(x) \quad \text { for all } x \in C .
$$

This theorem is at the base of the results that follow. For that reason it seems worthwhile to have a purely analytical proof; other proofs have been given by Godement [6, Théorème $\mathrm{A}$ ] and Segal [13, Theorem 3.8].

COROLlaRY. Let $\boldsymbol{f}(\mathbf{x}), \boldsymbol{g}(\mathbf{x})$, be in $L^{1}$. If $f(x)$ vanishes outside some compact set and the zeros of $g(x)$ are all interior to the set of zeros of $f(x)$, then there is a function $h(x) \in L^{1}$ such that

$$
f(x)=g(x) * h(x) .
$$

I. Closed ideals in $L^{1}$ AND their homomorphisms

LEMMA 1.1.1. If $f \in L^{1}$ is such that $f(e) \neq 0$, then every solution $\phi_{0} \in L^{\infty}$ of the integral equation

$$
\boldsymbol{f} * \boldsymbol{\phi}_{0}{ }^{*}(\mathbf{x})=0
$$

has the following property:

$$
\text { ess. } \sup \left|\boldsymbol{\gamma}+\boldsymbol{\phi}_{0}(\boldsymbol{x})\right|>|\boldsymbol{\gamma}| \text {, }
$$

for any constant $\gamma$, unless $\phi_{0}$ is zero almost everywhere.

Consider the function $m^{-1}(S) S(x)^{2}$. If $S$ is small, we have, by the corollary above since $f(x) \neq 0$ in some neighborhood of $e$, 


$$
m^{-1}(S) S(x)^{2}=f * h(x),
$$

so that

$$
m^{-1}(S) S(x)^{2} * \phi_{0}^{*}(x)=0 .
$$

Now

$$
m^{-1}(S) S(x)^{2} *\left|\gamma+\phi_{0}(x)\right|^{2} \leqq \text { ess. sup }\left|\gamma+\phi_{0}(x)\right|^{2} .
$$

The left-hand side may be written $\left({ }^{7}\right)$

$$
\begin{aligned}
m^{-1}(S) S(x)^{2} *\left\{|\boldsymbol{\gamma}|^{2}\right. & \left.+2 R\left[\gamma^{\circ} \boldsymbol{\phi}_{0}(\mathbf{x})\right]+\left|\boldsymbol{\phi}_{0}(\mathbf{x})\right|^{2}\right\} \\
& =|\boldsymbol{\gamma}|^{2}+2 R\left[\boldsymbol{\gamma}^{\cdot} m^{-1}(S) \mathbf{S}(\mathbf{x})^{2} * \boldsymbol{\phi}_{0}\right]+m^{-1}(S) \mathbf{S}(\mathbf{x})^{2} *\left|\boldsymbol{\phi}_{0}\right|^{2} .
\end{aligned}
$$

But the middle term vanishes and hence, unless $\phi_{0}$ is zero almost everywhere,

$$
|\gamma|^{2}<\text { ess. sup }\left|\gamma+\phi_{0}(x)\right|^{2}
$$

which was to be proved.

Lemma 1.1.2. If $f \in L^{1}$ is such that $f(e)=0$, then the integral equation

$$
\boldsymbol{f} * \boldsymbol{\phi}^{*}(\mathrm{x})=1
$$

has no solution $\phi \in L^{\infty}$.

Suppose there is a $\phi \in L^{\infty}$ such that

$$
\int f(y) \phi^{\bullet}\left(x^{-1} y\right) d y=1 \quad \text { for all } x \in G .
$$

Multiply both sides by $m^{-1}(S) S(x)^{2}$ and integrate with respect to $\mathbf{x}$ :

$$
\int m^{-1}(S) S(x)^{2} d x \int f(y) \phi^{\cdot}\left(x^{-1} y\right) d y=1
$$

or

$$
\int \boldsymbol{f}(\mathbf{y}) d \mathbf{y} \int m^{-1}(S) S(\mathbf{x})^{2} \boldsymbol{\phi}^{\bullet}\left(\mathbf{x}^{-1} \mathbf{y}\right) d \mathbf{x}=1 .
$$

Since $\int f(y) d y=0$, the left-hand side is equal to

$$
\begin{aligned}
\int f(y) d y \int m^{-1}(S) S(x)^{2}\left\{\phi^{\cdot}\right. & \left.\left(x^{-1} \mathbf{y}\right)-\phi^{\cdot}(\mathbf{x})\right\} d x \\
& =\int f(y) d y \int m^{-1}(S)\left\{S\left(y^{-1} \mathbf{x}\right)^{2}-S(x)^{2}\right\} \phi^{\cdot}(\mathbf{x}) d x .
\end{aligned}
$$

Given $\epsilon>0$, there is a compact set $C \subset G$ such that

(7) $R[z]=$ real part of $z$. 


$$
\int_{\mathcal{C}^{c}}|\boldsymbol{f}(\boldsymbol{y})| d \boldsymbol{y}<\epsilon
$$

Furthermore, for any $y \in C$,

$$
m^{-1}(S)\left\|S\left(y^{-1} \mathbf{x}\right)^{2}-\mathbf{S}(\mathbf{x})^{2}\right\|_{1}<\epsilon,
$$

provided $S$ is small, by property (ii'), p. 405. Thus the last repeated integral is in absolute value smaller than $\epsilon 2\|\boldsymbol{\phi}\|_{\infty}+\|\boldsymbol{f}\|_{1} \epsilon\|\boldsymbol{\phi}\|_{\infty}$. Since this may be made arbitrarily small, no solution $\phi \in L^{\infty}$ can exist.

Given a function $\boldsymbol{f} \in L^{1}$, denote by $(\boldsymbol{f})$ the closed ideal generated by $\boldsymbol{f}$, i.e., the smallest closed ideal in $L^{1}$ containing $f$. It may be defined explicitly as the closure of the set of all linear combinations of "translates" of $\boldsymbol{f}(\mathbf{x})$ :

$$
\sum_{n=1}^{N} a_{n} f\left(y_{n} \mathbf{x}\right)
$$

where $a_{1}, a_{2}, \cdots, a_{N}$ are arbitrary complex numbers, $y_{1}, y_{2}, \cdots, y_{N}$ are any elements of $G$, and $N$ is any positive integer (cf. p. 403).

Define now the ideal $(\boldsymbol{f})_{e}$ as the closure of the set of all linear combinations

$$
\sum_{n=1}^{N} b_{n} f\left(y_{n} x\right)
$$

where the coefficients $b_{n}$ satisfy the condition

$$
\sum_{n=1}^{N} b_{n}=0 .
$$

The reason for the notation will appear later.

REMARK. It is important to observe that $(f)_{e}$ may also be defined as the closure of the set of all linear combinations

$$
\sum_{n=1}^{N} a_{n}\left\{f\left(y_{n} \mathbf{x}\right)-\boldsymbol{f}(\mathbf{x})\right\}
$$

with arbitrary coefficients $a_{n}$.

Theorem 1.1. For any $\boldsymbol{f}(\mathrm{x}) \in L^{1}$

$$
\inf \int\left|\boldsymbol{f}(\mathbf{x})-\sum_{n=1}^{N} b_{n} f\left(y_{n} \mathbf{x}\right)\right| d \mathbf{x}=\left|\int \boldsymbol{f}(\mathbf{x}) d \mathbf{x}\right|,
$$

where $N$ ranges over all positive integers, $b_{1}, b_{2}, \cdots, b_{N}$ range over all complex numbers satisfying $\sum_{n=1}^{N} b_{n}=0$, and $y_{1}, y_{2}, \cdots, y_{N}$ over the elements of $G$.

(If the equality sign is replaced by " $\geqq$ ", the statement becomes trivial.) Making use of the definition of the ideal $(f)_{e}$, the theorem may be stated 
concisely thus:

$$
\operatorname{dist}\left\{\boldsymbol{f},(\boldsymbol{f})_{e}\right\}=|f(e)| .
$$

The proof is an application of the distance theorem (p. 402): we have

$$
\operatorname{dist}\left\{f,(f)_{e}\right\}=1 / \min \|\phi\|_{\infty},
$$

where $\phi$ ranges over all functions in $L^{\infty}$ satisfying

$$
\begin{gathered}
\int f(y) \phi^{\cdot}(y) d y=1, \\
\int\{f(x y)-f(y)\} \phi^{\cdot}(y) d y=0 \quad(x \in G)
\end{gathered}
$$

(here the remark on p. 411 is used). If no such $\phi$ exists, then $f \in(f)_{e}$.

Thus we have to investigate the solutions of the integral equation

$$
f * \phi^{*}(\mathbf{x})=1 .
$$

Suppose first that $\boldsymbol{\gamma}=\int \boldsymbol{f}(\mathbf{x}) d \mathbf{x} \neq 0$. Then every solution may be written in the form

$$
\phi(x)=1 / \boldsymbol{\gamma}^{\bullet}+\phi_{0}(\mathbf{x}),
$$

where $\phi_{0}$ satisfies $\boldsymbol{f} * \boldsymbol{\phi}_{0}^{*}(x)=0$. By Lemma 1.1.1 the function $\phi(x)=1 / \boldsymbol{\gamma}^{\bullet}$ is the solution with the smallest norm and hence

$$
\text { dist, }\left\{\boldsymbol{f},(\boldsymbol{f})_{e}\right\}=|f(e)| \text {. }
$$

If $\int \boldsymbol{f}(\mathbf{x}) d \mathbf{x}=0$, then, by Lemma 1.1 .2 , there is no $\phi \in L^{\infty}$ satisfying $f * \boldsymbol{\phi}^{*}(\boldsymbol{x})=1$ and hence $\boldsymbol{f} \in(\boldsymbol{f})_{e}$. Thus the proof of the theorem is complete.

Theorem 1.1 may be generalized. Let $I$ be the closed ideal generated by the functions $f, \boldsymbol{g}, \cdots$, i.e., the smallest closed ideal containing $(\boldsymbol{f}),(\boldsymbol{g}), \cdots$; we express this by writing

$$
I=(\boldsymbol{f}, \boldsymbol{g}, \cdots) .
$$

We define now the ideal $I_{\theta}$ as the smallest closed ideal containing $(\boldsymbol{f})_{e},(\boldsymbol{g})_{e}, \cdots$ and write

$$
I_{e}=(f, \xi, \cdots)_{e} .
$$

Thus $I_{e}$ is the closure of the set of all finite sums of certain linear combinations (see p. 411) of the translates of $f, \xi, \cdots$, respectively.

Then for any $k \in I$

$$
\operatorname{dist}\left\{k, I_{e}\right\}=|k(e)| \text {. }
$$

Indeed, since $k \in I$, there are, for any $\epsilon>0$, among the generating functions $f, \xi, \cdots$, a finite number, say $f_{1}, f_{2}, \cdots, f_{R}$ such that the function 


$$
k_{\epsilon}(x)=\sum_{r=1}^{R} \sum_{s=1}^{S r} a_{r s} f_{r}\left(y_{r s} \mathrm{x}\right)
$$

(where the choice of the coefficients $a_{r s}$ and the elements $y_{r s} \in G$ depends on $\epsilon$ ) satisfies the condition

$$
\left\|k(x)-k_{e}(x)\right\|_{1}<\epsilon .
$$

Consider now the function $k_{\epsilon}$ and the ideal $\left(k_{\epsilon}\right)_{e}$ : by Theorem 1.1

$$
\operatorname{dist}\left\{k_{\epsilon},\left(k_{\epsilon}\right)_{e}\right\}=\left|\int k_{\epsilon}(\mathbf{x}) d \mathbf{x}\right| \text {. }
$$

From the definition of $I_{\theta}$ it is clear that

$$
\left(k_{\epsilon}\right), \subset I_{\theta}
$$

and since for all $h \in I_{e}$

$$
\int\left|k_{\epsilon}(\mathrm{x})-\boldsymbol{h}(\mathrm{x})\right| d \mathbf{x} \geqq\left|\int k_{\epsilon}(\mathrm{x}) d \mathbf{x}\right|,
$$

it follows that

$$
\operatorname{dist}\left\{k_{\epsilon}, I_{e}\right\}=\left|\int k_{\epsilon}(\mathbf{x}) d \mathbf{x}\right| .
$$

This implies that

$$
\begin{aligned}
\operatorname{dist}\left\{k, I_{e}\right\} & \leqq\left\|k-k_{\epsilon}\right\|_{1}+\left|\int k_{\epsilon}(\mathbf{x}) d \mathbf{x}\right| \\
& \leqq \epsilon+\left|\int k(\mathrm{x}) d \mathbf{x}\right|+\epsilon .
\end{aligned}
$$

Now the inequality dist $\left\{k, I_{e}\right\} \geqq\left|\int k(x) d x\right|$ is again trivial and hence, since $\epsilon>0$ is arbitrary, the result is proved.

We proceed now to the final generalization of Theorem 1.1.

If $I=(f)$, we define the ideal $I_{x}=(f)_{x}$ as the closure of the set of all linear combinations

$$
\sum_{n=1}^{N} b_{n}\left(y_{n}, x\right) \cdot f\left(y_{n} x\right)
$$

where $x$ is a fixed element of $G, N$ is any positive integer, $y_{n} \in G(1 \leqq n \leqq N)$, and the coefficients satisfy the condition

$$
\sum_{n=1}^{N} b_{n}=0
$$


If $I=(f, g, \cdots)$, we define $I_{x}=(f, \xi, \cdots)_{x}$ as the smallest closed ideal containing $(f)_{x},(\mathcal{g})_{x}, \cdots$. We have now (in the notation of p. 404):

$$
\operatorname{dist}\left\{\boldsymbol{k}(\mathbf{x}), \boldsymbol{I}_{x}\right\}=\operatorname{dist}\left\{\boldsymbol{k}(\mathbf{x})(\mathbf{x}, x)^{\bullet}, \boldsymbol{I}_{x}(\mathbf{x}, x)^{\cdot}\right\} .
$$

But the ideal $I_{x}(\mathrm{x}, x)^{\bullet}$ bears to the ideal $I(\mathrm{x}, x)^{\bullet}$ the relation

$$
I_{x}(\mathrm{x}, x)^{\bullet}=\left(\mathrm{f}(\mathrm{x})(\mathrm{x}, x)^{\bullet}, \mathrm{g}(\mathrm{x})(\mathrm{x}, x)^{\bullet}, \cdots\right)_{e}=I(\mathrm{x}, x)_{e},
$$

and hence we may apply the first generalization of Theorem 1.1 to the function $k(x)(x, x)^{\bullet} \in I(x, x)^{\bullet}$ and the ideal $I(x, x)^{*}$, so that we finally obtain

$$
\operatorname{dist}\left\{k, I_{x}\right\}=|k(x)| \text {. }
$$

The results obtained may now be summarized as follows:

Theorem 1.2. Let I be a closed ideal in $L^{1}$. Define, for a fixed $x \in G$, the ideal $I_{x} \subset I$ as above. Then for any function $f \in I$

$$
\operatorname{dist}\left\{\boldsymbol{f}, \boldsymbol{I}_{x}\right\}=|f(x)| \text {. }
$$

Hence $I_{x}$ is the kernel of the homomorphism

$$
\boldsymbol{f} \rightarrow f(x)
$$

of $I$ into $C$, the field of complex numbers. Thus $I_{x}$ is a maximal ideal of $I$ or coincides with $I$ according as $x \in \mathcal{C} Z_{I}$ or $x \in Z_{I}$. Moreover, if the quotientalgebra $I / I_{x}$ is considered as a Banach algebra $\left({ }^{8}\right)$, then the isomorphism

$$
I / I_{x} \cong C
$$

preserves the norm.

Conversely: given a continuous homomorphism

$$
\boldsymbol{f} \rightarrow \mu(\boldsymbol{f})
$$

of I upon $C$, then

$$
\mu(f)=\int f(x)\left(x, x_{\mu}\right) \cdot d x,
$$

where $x_{\mu} \in G$ is a uniquely determined element of $\mathcal{C} Z_{I}$.

To establish the converse, let $s$ be any (fixed) element of $G$. It is proved in $[6, \mathrm{p} .122]$ that

$$
\left.\mu f\left(s^{-1} x\right)\right)=\left(s, x_{\mu}\right)^{\cdot} \mu(f),
$$

where $x_{\mu}$ is a uniquely determined element of $G$.

Consider now the ideal $I_{x_{\mu}}$. It is the kernel of the (continuous) homomorphism

(8) See, e.g. [8, Theorem 22.11.4]. 


$$
f \rightarrow \int f(x)\left(x, x_{\mu}\right) \cdot d x
$$

Let $I_{\mu}$ be the kernel of the given homomorphism $f \rightarrow \mu(f)$. If we can prove that $I_{x_{\mu}} \subset I_{\mu}$, it will follow that $I_{x_{\mu}}=I_{\mu}$ and hence that $\mu(f)=\int f(\mathrm{x})\left(\mathrm{x}, x_{\mu}\right)^{*} d \mathbf{x}$.

Suppose there is a function $g \in I_{x_{\mu}}$ which is not in $I_{\mu}$. Then $\mu(\xi) \neq 0$ and, for all $s \in G$,

$$
\mu\left(\boldsymbol{g}\left(\mathbf{s}^{-1} \mathbf{x}\right)\right)=\left(\mathbf{s}, x_{\mu}\right)^{\bullet} \mu(\boldsymbol{g}),
$$

while

$$
\int \boldsymbol{g}(\mathbf{x})\left(\mathrm{x}, x_{\mu}\right) \cdot d \mathbf{x}=0 .
$$

Now $\mu(f)$, considered as a bounded linear functional on $I$, may be extended to the whole space $L^{1}$, by the complex analogue of the Hahn-Banach theorem [8, Theorems 2.9.2 and 2.9.5]. Hence

$$
\mu(\boldsymbol{f})=\int \boldsymbol{f}(\mathbf{x}) \dot{\boldsymbol{\phi}_{\mu}}(\mathbf{x}) d \mathbf{x} \quad \quad\left(\boldsymbol{\phi}_{\mu} \in L^{\infty}\right) .
$$

We have therefore for the function $\boldsymbol{g}(\mathbf{x})$ :

$$
\begin{aligned}
& \int \boldsymbol{g}\left(\mathbf{s}^{-1} \mathbf{x}\right) \dot{\phi_{\mu}}(\mathbf{x}) d \mathbf{x}=\left(\mathrm{s}, x_{\mu}\right)^{\cdot} \mu(\boldsymbol{g}) \quad \text { for all } \mathrm{s} \in G, \\
& \int \boldsymbol{g}(\mathrm{x})\left(\mathrm{x}, x_{\mu}\right)^{\cdot} d \mathbf{x}=0 .
\end{aligned}
$$

If we write this in a slightly different form and apply Lemma 1.1.2, we see that it is impossible. This completes the proof.

THEOREM 1.3. If the group $G^{\prime}$ is a homomorphic image of $G$, then the $L^{1}$-algebra on $G^{\prime}$ is a homomorphic image of the $L^{1}$-algebra on $G$. The kernel $I_{0}$ of this homomorphism consists of all functions in $L^{1}(G)$ whose Fourier transforms vanish on the dual group $G^{\prime} \subset G$, and the isomorphism

$$
L^{1}(G) / I_{0} \cong L^{1}\left(G^{\prime}\right)
$$

preserves the norm (the measure on $G^{\prime}$ being properly normalized).

For any $f(x) \in L^{1}(G)$, define

$$
T(f(x))=f^{\prime}\left(x^{\prime}\right)=\int_{g} f(x y) d y,
$$

where the closed subgroup $g \subset G$ is such that $G^{\prime}=G / g\left[14\right.$, p. 11], and $x^{\prime}$ denotes the coset $\mathbf{x} \boldsymbol{g}$. Then $f^{\prime}\left(x^{\prime}\right) \in L^{1}\left(G^{\prime}\right)$ and (with proper normalization of 
the measure)

$$
\int_{G} f(x) d x=\int_{G^{\prime}} f^{\prime}\left(\mathbf{x}^{\prime}\right) d \mathbf{x}^{\prime} .
$$

This result (immediate for $G=R^{1}$ ) is proved in [1]. We have also

$$
\|f\|_{1} \geqq\left\|f^{\prime}\right\|_{1} .
$$

Furthermore, since $G^{\prime}=G / \mathcal{g}$, the dual group $G^{\prime}$ is contained in $G$ and $\left(y, x^{\prime}\right)=1$ whenever $y \in g, x^{\prime} \in G^{\prime}$ (cf. [14, pp. 108-109]). Thus for $x=x^{\prime} \in G^{\prime}$,

$$
\begin{aligned}
\int_{G} f(x)\left(x, x^{\prime}\right) \cdot d x & =\int_{G^{\prime}} d x^{\prime} \int_{\xi} f(x y)\left(x y, x^{\prime}\right) \cdot d y \\
& =\int_{G^{\prime}} d x^{\prime}\left(x^{\prime}, x^{\prime}\right) \cdot \int_{\xi} f(x y) d y \\
& =\int_{G^{\prime}} f^{\prime}\left(x^{\prime}\right)\left(x^{\prime}, x^{\prime}\right) \cdot d x^{\prime} .
\end{aligned}
$$

Since a function in $L^{1}\left(G^{\prime}\right)$ is entirely determined by its Fourier transform, it follows that $T(\boldsymbol{f}(\mathrm{x}))$ is determined by the values of $f(x)$ for $x=x^{\prime} \in G^{\prime}$. Hence we have

$$
T\left(f_{1} * f_{2}\right)=T\left(f_{1}\right) * T\left(f_{2}\right),
$$

i.e., $T$ is a homomorphism of $L^{1}(G)$ into $L^{1}\left(G^{\prime}\right)$.

The kernel of this homomorphism is the closed ideal $I_{0}$ consisting of all functions in $L^{1}(G)$ whose Fourier transforms vanish on $G^{\prime} C G$.

We have

$$
\inf \left\|f(x)-f_{0}(x)\right\|_{1} \geqq\left\|f^{\prime}\left(x^{\prime}\right)\right\|_{1}
$$

To establish the opposite inequality, we use the distance theorem (cf. p. 402):

$$
\operatorname{dist}\left\{f, I_{0}\right\}=1 / \min \|\phi\|_{\infty}
$$

where

$$
\phi \perp I_{0}, \quad \int f(x) \phi^{\bullet}(x) d x=1 .
$$

Lemma. Any $\phi \perp I_{0}$ has the property that

$$
\boldsymbol{\phi}(\mathbf{s x})=\boldsymbol{\phi}(\mathbf{x})
$$

almost everywhere (and conversely).

For any $k(x) \in L^{1}(G), k(s x)-k(x) \in I_{0}$, i.e., 


$$
[k(s \mathrm{x})-k(\mathrm{x})] * \phi^{*}(\mathrm{x})=0
$$

or

$$
k(x) *\left[\phi^{*}\left(\mathbf{s}^{-1} \mathbf{x}\right)-\phi^{*}(\mathbf{x})\right]=0 .
$$

Since $k \in L^{1}(G)$ and $s \in g$ are arbitrary, the assertion follows (the converse follows from the fact that $f(x) * \phi^{*}=f^{\prime}\left(x^{\prime}\right) * \phi^{\prime *}$-cf. below).

Thus the function $\phi \perp I_{0}$ defines a function $\phi^{\prime}\left(x^{\prime}\right)$ on $G^{\prime}$ and we have

$$
1=\int \boldsymbol{f}(\mathbf{x}) \boldsymbol{\phi}^{\cdot}(\mathbf{x}) d \mathbf{x}=\int \mathbf{f}^{\prime}\left(\mathbf{x}^{\prime}\right) \boldsymbol{\phi}^{\prime} \cdot\left(\mathbf{x}^{\prime}\right) d \mathbf{x}^{\prime} .
$$

Hence

$$
\left\|\phi^{\prime}\right\|_{\infty}\left\|f^{\prime}\right\|_{1} \geqq 1
$$

and thus

$$
\|\phi(x)\|_{\infty} \geqq 1 /\left\|f^{\prime}\right\|_{1} .
$$

This gives the opposite inequality.

Thus the Banach quotient-algebra $L^{1}(G) / I_{0}$ is not only algebraically isomorphic, but also isometric with the image of $L^{1}(G)$ in $L^{1}\left(G^{\prime}\right)$ under the homomorphism $T$. It remains to show that this image is $L^{1}\left(G^{\prime}\right)$ itself.

Since $L^{1}(G) / I_{0}$ is complete, it suffices to show that the functions $T(f)$ are dense in $L^{1}\left(G^{\prime}\right)$. But this follows from a proof given by A. Weil [14, pp. 42-43]. Thus the theorem is established.

\section{A generalization of WiENER's THEOREM, With applications}

By Wiener's theorem is meant Theorem IV in [15]. It was extended by Godement [6, p. 125] to locally compact abelian groups and reads:

Let $I$ be a closed ideal in $L^{1}$. In order that $I=L^{1}$, it is necessary and sufficient that for every $x_{0} \in G$ there exist an $f(x) \in I$ such that $f\left(x_{0}\right) \neq 0$.

In the course of the proof of Wiener's theorem a result is established which may be stated as follows:

An ideal I contains all functions $\boldsymbol{f}_{C}(\mathbf{x})$ whose Fourier transforms vanish outside some compact set $C \subset C Z_{I}$.

(Actually this is stated here in a slightly more general form which is precisely what we shall need. The proof is that of Godement.)

For any $x_{\iota} \in C$ there is a function $f_{b} \in I$ such that $\left|f_{l}(x)\right| \geqq 1$ in some small neighborhood $x_{\mathfrak{}} S_{\downarrow}$ of $x_{\mathfrak{}}$. Since $C$ is compact, it may be covered by a finite number of those neighborhoods, $x_{r} S_{r}(1 \leqq r \leqq N)$. Then the Fourier transform of the function $\sum_{r=1}^{N} \boldsymbol{f}_{r}(\mathrm{x}) * \boldsymbol{f}_{r}^{*}(\mathrm{x}) \in I$ is strictly positive on $C$. If we now apply the corollary, p. 409 , to $f_{C}(x)$ and this function, the result follows.

We shall suppose from now on that $G$ has a denumerable fundamental system of neighborhoods of the identity (but cf. Appendix). We may then 
choose in $G$ a fundamental sequence $\left(S_{n}\right), n \geqq 1$, of compact, symmetrical neighborhoods of $e$ such that $S_{n+1} \subset S_{n}$. Then every subsequence $\left(S_{n_{r}}\right), r \geqq 1$, will still be a fundamental system.

Lemma 2.1.1. For any function $\phi \in L^{\infty}$ there exists (at least) one constant $M$ such that, for a certain subsequence $\left(n_{r}\right)$,

$$
m^{-1}\left(S_{n_{r}}\right) S_{n_{r}}(\mathbf{x})^{2} * \phi \rightarrow M
$$$$
(r \rightarrow \infty)
$$

for all $\mathbf{x} \in G$.

Since the numbers $m^{-1}\left(S_{n}\right) \int \boldsymbol{S}_{n}(\boldsymbol{y})^{2} \boldsymbol{\phi}(\boldsymbol{y}) d \boldsymbol{y}$ are all bounded, in absolute value, by $\|\boldsymbol{\phi}\|_{\infty}$, there exists a subsequence $\left(n_{r}\right)$ such that $m^{-1}\left(S_{n_{r}}\right) \int \boldsymbol{S}_{n_{r}}(\boldsymbol{y})^{2}$ $\cdot \boldsymbol{\phi}(\boldsymbol{y}) d y$ tends to a limit, $M$, say, as $r \rightarrow \infty$.

We want to show that for any (fixed) $x \in G, m^{-1}\left(S_{n_{r}}\right) S_{n_{r}}(x)^{2} * \phi \rightarrow M$ $(r \rightarrow \infty)$. It will be sufficient to prove that

$$
m^{-1}\left(S_{n_{r}}\right) \int S_{n_{r}}(y)^{2}\left\{\phi\left(x y^{-1}\right)-\phi(y)\right\} d y \rightarrow 0 \quad(r \rightarrow \infty)
$$

for any (fixed) $x \in G$. Now the integral may be written

$$
m^{-1}\left(S_{n_{r}}\right) \int\left\{S_{n_{r}}\left(\mathbf{x}^{-1} \mathbf{y}\right)^{2}-S_{n_{r}}(y)^{2}\right\} \boldsymbol{\phi}(y) d y
$$

and this, in absolute value, will be smaller than $\epsilon\|\phi\|_{\infty}$, by property (ii), p. 404 , if $n_{r}$ is sufficiently large. This completes the proof.

Lemma 2.1.2. Suppose that $\phi \perp I$ and that the identity $e \in G$ is not a limiting point of $Z_{I}$. Then, if the neighborhood $S$ is small $\left(S^{2} \cap Z_{I} \cap \mathcal{C}\{e\}=\varnothing\right)$,

$$
m^{-1}(S) S(\mathbf{x})^{2} * \phi=\text { const., }
$$

the constant being independent of $S$ (subject to the above condition).

REMARK. This is of course trivial if $G$ is compact, since then we may take $S=\{e\}$. See also the Appendix at the end of this paper.

Take a neighborhood $S_{N}$ of the fundamental sequence of neighborhoods, so small that $S^{2} S_{N} \cap Z_{I} \cap \mathcal{C}\{e\}=\varnothing$. Then the Fourier transform

$$
S * S S_{N}(x)-S * S S_{n}(x), \quad n>N,
$$

vanishes outside the set $S^{2} S_{N} \cap C S_{n}$ (cf. p. 405; it may be helpful to plot a figure for the case $G=R^{1}$ ). Applying the result on p. 417 , we have

$$
S(x) \cdot S S_{N}(x)-S(x) \cdot S S_{n}(x) \in I .
$$

It is our first aim to prove that

$$
S(x) \cdot S S_{N}(x)-S(x)^{2} \in I .
$$


Indeed, we have

$$
\begin{aligned}
S(x) \cdot S S_{N}(x)-S(x)^{2}= & \left\{S(x) \cdot S S_{N}(x)-S(x) \cdot S S_{n}(x)\right\} \\
& +\left\{S(x) \cdot S S_{n}(x)-S(x)^{2}\right\} .
\end{aligned}
$$

The first part is in $I$ (see above); the second is, in $L^{1}$-norm, less than $\left\{m\left(S S_{n}\right)-m(S)\right\}^{1 / 2} m^{1 / 2}(S)$ (cf. property (iii), p. 405). But this can be made arbitrarily small, by taking $n$ sufficiently large $\left({ }^{9}\right)$, and thus the partial result is proved.

On the other hand, if we take $S_{n} \subset S$, then

$$
m^{-1}(S) \mathbf{S}(\mathbf{x}) \cdot \mathbf{S} \boldsymbol{S}_{N}(\mathbf{x})-m^{-1}\left(S_{n}\right) \boldsymbol{S}_{n}(\mathbf{x}) \cdot \boldsymbol{S}_{n} \boldsymbol{S}_{N}(\mathbf{x}) \in I
$$

(cf. p. 405) the reader may again find it helpful to plot a graph of the Fourier transforms for the case $G=R^{1}$ ).

But

$$
m^{-1}\left(S_{n}\right) S_{n}(\mathbf{x}) \cdot S_{n} S_{N}(\mathbf{x})-m^{-1}\left(S_{n}\right) S_{n}(\mathbf{x})^{2} \in I
$$

(set $S=S_{n}$ in the partial result just proved).

Hence finally

$$
\dot{m}^{-1}(S) \mathbf{S}(\mathbf{x})^{2}-m^{-1}\left(S_{n}\right) S_{n}(\mathbf{x})^{2} \in I
$$

for all $S_{n} \subset S$, i.e.,

$$
\left\{m^{-1}(S) S(\mathbf{x})^{2}-m^{-1}\left(S_{n}\right) S_{n}(\mathbf{x})^{2}\right\} * \phi^{*}=0,
$$

$\phi$ being the function of the hypothesis. Hence we have

$$
m^{-1}(S) S(x)^{2} * \boldsymbol{\phi}=m^{-1}\left(S_{n}\right) S_{n}(x)^{2} \Pi \phi .
$$

Let now $\left(n_{r}\right)$ be a subsequence such that

$$
m^{-1}\left(S_{n_{r}} S_{n_{r}}(\mathbf{x})^{2} * \phi \rightarrow M\right.
$$$$
(r \rightarrow \infty)
$$

(Lemma 2.1.1). Since the left-hand side of the above equation is independent of $n$, it follows that

$$
m^{-1}(S) S(x)^{2} * \phi=M=\text { const., }
$$

which proves the lemma. It also entails that $M$ is unique. In this case we write $M\{\boldsymbol{\phi}(\mathbf{x})\}$ instead of $M$ and call it the mean value of $\boldsymbol{\phi}(\mathbf{x})$.

This terminology is justified by the fact that, if $\alpha(x)$ is not only in $L^{\infty}$, but also almost periodic in the sense of von Neumann, then

$$
\lim _{n \rightarrow \infty} m^{-1}\left(S_{n}\right) \int S_{n}(\mathbf{x})^{2} \boldsymbol{\alpha}(\mathbf{x}) d \mathbf{x}
$$

(9) This follows from the regularity of the Haar measure (cf. [7]) and the fact that, for any open set $O$ containing $S$, the product $S S_{n}$ will be contained in $O$ for large $n$. 
exists and coincides with the usual mean value. Let $A$ be that mean value. Then, given $\epsilon>0$, there exist positive numbers $c_{1}, c_{2}, \cdots, c_{N}$, whose sum is 1 , and elements $y_{1}, y_{2}, \cdots, y_{N} \in G$ such that for all $x \in G$

$$
\left|c_{1} \boldsymbol{\alpha}\left(\boldsymbol{y}_{1} \mathbf{x}\right)+\cdots+c_{N} \boldsymbol{\alpha}\left(\boldsymbol{y}_{N} \mathbf{x}\right)-A\right|<\epsilon .
$$

We may write the difference

$$
m^{-1}\left(S_{n}\right) \int \boldsymbol{S}_{n}(\mathbf{x})^{2} \boldsymbol{\alpha}(\mathbf{x}) d \mathbf{x}-A
$$

in the form

$$
\begin{aligned}
\sum_{r=1}^{N} c_{r} m^{-1}\left(S_{n}\right) \int S_{n}(\mathbf{x})^{2}\left\{\boldsymbol{\alpha}(\mathrm{x})-\boldsymbol{\alpha}\left(\mathrm{y}_{r} \mathrm{x}\right)\right\} d \mathrm{x} & \\
& +m^{-1}\left(S_{n}\right) \int S_{n}(\mathrm{x})^{2}\left\{\sum_{r=1}^{N} c_{r} \boldsymbol{\alpha}\left(y_{r} \mathrm{x}\right)-A\right\} d \mathrm{x} .
\end{aligned}
$$

But each term of the first sum tends to 0 , as $n \rightarrow \infty$, by property (ii), p. 404, and the second term is in absolute value less than $\epsilon$, for every $n$. Since $\epsilon>0$ is arbitrary, the result is proved.

From Lemma 2.1.2 there follows immediately a general result:

Theorem 2.1. Let $I$ be a closed ideal in $L^{1}$ and suppose that $x_{1} \in G$ is not a limiting point of $Z_{I}$. Suppose that $\phi \perp I$. Then the mean value of $\phi(x)\left(x, x_{1}\right)^{\circ}$ exists and, if $S$ is sufficiently small $\left(x_{1} S^{2} \cap Z_{I} \cap \complement\left\{x_{1}\right\}=\varnothing\right)$,

$$
m^{-1}(S) \mathbf{S}(\mathbf{x})^{2} *\left[\boldsymbol{\phi}(\mathbf{x})\left(\mathbf{x}, x_{1}\right)^{\bullet}\right]=M\left\{\boldsymbol{\phi}(\mathbf{x})\left(\mathbf{x}, x_{1}\right)^{\bullet}\right\} .
$$

Indeed, we may reduce this case to Lemma 2.1 .2 by writing $\phi(x)\left(x, x_{1}\right)^{\circ}$ $\perp I\left(x, x_{1}\right)$ ", the right-hand side denoting the "rotated" ideal (cf. p. 404).

Now we are ready to prove a generalization of Wiener's theorem (for groups $G$ whose dual groups have a denumerable fundamental system of neighborhoods of the identity; cf. however the Appendix):

TheOREM 2.2. Let $I$ be a closed ideal in $L^{1}$, with co-spectrum $Z_{I}$ (p. 404). If the function $k(x) \in L^{1}$ is such that its Fourier transform $k(x)$ satisfies the following conditions:

(i) $Z_{k} \supset Z_{I}\left(Z_{k}\right.$ denotes the set of zeros of $\left.k(x)\right)$;

(ii) $\mathcal{F}\left(Z_{k}\right) \cap \mathcal{F}\left(Z_{I}\right)$ is denumerable $(\mathcal{F}(Z)$ denotes the frontier of $Z$ ), then $k(\mathbf{x}) \in I$.

This theorem was first proved, for the case $G=R^{1}$, by S. Mandelbrojt and S. Agmon [11, Théorème II], making use of Carleman's analytic functions $[3$, p. 75]. The reader will see for himself the connection between their proof and that given here. 
To prove the theorem, it suffices to show (cf. the distance theorem p. 402) that whenever $\phi \perp I$ then $\phi \perp k$.

Consider a $\phi \perp I$ and define the function $\phi_{1}(\mathbf{x})$ by $\phi_{1}(\mathbf{x})=k^{*}(\mathbf{x}) * \boldsymbol{\phi}(\mathbf{x})$. If we can prove that $\phi_{1} \perp L^{1}$, then it will follow that $k * \phi^{*}(x)=0$, and $\phi \perp k$.

Let $I_{1}$ be the set of all $f \in L^{1}$ such that $f * \phi_{1}^{*}=0$; this is a closed ideal. We shall prove:

$$
\begin{aligned}
& Z_{I_{1}} \subset \mathcal{F}\left(Z_{k}\right) \cap \mathcal{F}\left(Z_{I}\right), \\
& Z_{I_{1}} \text { has no isolated points. }
\end{aligned}
$$

From (1) it follows, under the hypothesis of the theorem, that $Z_{I_{1}}$ is denumerable, and from (2) that it is perfect. This implies that $Z_{I_{1}}=\varnothing$, and hence (Wiener's theorem) $I_{1}=L^{1}$. Thus the theorem will be proved if we can establish (1) and (2).

(1) Since $g * \phi^{*}=0$ for any $g \in I$, we have

$$
g * \phi_{1}^{*}=g * k * \phi^{*}=k * g * \phi^{*}=0 .
$$

Thus $\phi_{1} \perp I$, i.e., $I \subset I_{1}$, and hence $Z_{I_{1}} \subset Z_{I}$. Now we shall show that no interior point of $Z_{k}$ can belong to $Z_{I_{1}}$.

Indeed, if $x_{0}$ is an interior point of $Z_{k}$, let $S$ be so small that $x_{0} S^{2} \subset Z_{k}$, and consider the function $\left(x, x_{0}\right) S(x)^{2}$. This function is in $I_{1}$, since $\left[\left(x, x_{0}\right) S(x)^{2}\right]$ $* \phi_{1}^{*}=\left[\left(x, x_{0}\right) S(x)^{2}\right] * k * \phi^{*}=0$ (observe that the Fourier transform of $\left[\left(\mathrm{x}, x_{0}\right) S(\mathrm{x})^{2}\right] * k$ vanishes identically). Thus $x_{0}$ is not in $Z_{I_{1}}$ (cf. [11, Lemme I]).

Therefore $Z_{I_{1}}$ is in $Z_{I}$ and contains no point interior to $Z_{k}$. Since $Z_{I} \subset Z_{k}$, the only points that can possibly belong to $Z_{I_{1}}$ are those points of $\mathcal{F}\left(Z_{I}\right)$ which are in $\mathcal{F}\left(Z_{k}\right)$. Thus (1) is proved.

(2) Let us suppose that $x_{1} \in \mathcal{F}\left(Z_{I}\right) \cap \mathcal{F}\left(Z_{k}\right)$ is not a limiting point of $Z_{I_{1}}$. Consider first the case $x_{1}=e$. By Theorem 2.1

or

$$
m^{-1}(S) S(\mathrm{x})^{2} * \phi_{1}=M\left\{\phi_{1}\right\}
$$

$$
m^{-1}(S) S(x)^{2} * \phi_{1}^{*}=M \cdot\left\{\phi_{1}\right\},
$$

e.i., $m^{-1}(S) S(x)^{2} * k * \phi^{*}=M \cdot\left\{\phi_{1}\right\}$.

Take now $S=S_{n}$. By Lemma 2.1.1 there exists a subsequence $\left(n_{r}\right)$ such that $m^{-1}\left(S_{n_{r}}\right) S_{n_{r}}(x)^{2} * \phi^{*}$ tends (boundedly) to a constant $c(r \rightarrow \infty)$. But then the left-hand side of the last equation tends to $c \int k(\mathbf{x}) d \mathbf{x}=c \cdot k(e)=0$. Thus $M \cdot\left\{\phi_{1}\right\}=0$, i.e., $S(x)^{2} \in I_{1}$, and hence $e$ is not in $Z_{I_{1}}$ (see also Appendix).

The general case may be reduced to the preceding one by considering instead of $\phi_{1}(x)$ the function

$$
\phi_{1}(\mathrm{x})\left(\mathrm{x}, x_{1}\right)^{\bullet}=\left[k(\mathrm{x})\left(\mathrm{x}, x_{1}\right)^{\bullet}\right]^{*} *\left[\boldsymbol{\phi}(\mathrm{x})\left(\mathrm{x}, x_{1}\right)^{\bullet}\right] .
$$

The ideal corresponding to that function is just the "rotated" ideal (cf. 
p. 404) $I_{1}\left(x, x_{1}\right)^{\bullet}$, with co-spectrum $x_{1}^{-1} Z_{I_{1}}$, and the co-spectrum of $k(x)\left(x, x_{1}\right)^{\bullet}$ is $x_{1}^{-1} Z_{k}$. Thus, by the special case just proved, $e$ is not in $x_{1}^{-1} Z_{I_{1}}$, i.e., $x_{1}$ is not in $Z_{I_{1}}$, and the proof of (2) is complete.

REMARKs. Condition (i) of the theorem is obviously necessary as well; moreover, for compact groups it is also sufficient, condition (ii) being then superfluous, since the dual group of a compact group is discrete. Thus for compact groups $G$ there is a one-to-one correspondence between the closed ideals of $L^{1}$ and the subsets of $G$ which inverts the inclusion (cf. [10, p. 392]). On the other hand L. Schwartz has proved [12] that condition (i) alone is not sufficient in general, if $G$ is not compact. This shows the role of condition (ii).

The reader is referred to Mackey's survey [10, pp. 392-395] for a discussion of previous generalizations of Wiener's theorem. Besides the papers by Mandelbrojt-Agmon [11] and Godement [6], we mention here in particular those of Ditkin [5], Segal [13], and Kaplansky [9] $\left({ }^{10}\right)$.

We shall now apply the preceding results to study the functions $\phi \in L^{\infty}$ orthogonal to a given ideal $I$, when the co-spectrum $Z_{I}$ has a particularly simple structure.

If $Z_{I}$ is finite, then any $\boldsymbol{\phi} \perp I$ is a linear combination, with constant coefficients, of the characters of $G$ defined by the elements of $Z_{I}$.

Let $Z_{I}$ consist of $x_{1}, x_{2}, \cdots, x_{N}$. If $k(x)$ is any function in $L^{1}$, then (for $S$ suitably small)

$$
\boldsymbol{k}(\mathrm{x})-\boldsymbol{k}(\mathrm{x}) * \sum_{n=1}^{N}\left(\mathrm{x}, x_{n}\right) m^{-1}(S) \mathbf{S}(\mathbf{x})^{2}
$$

will be in $I$ (Theorem 2.2), i.e.,

$$
\left[k(\mathrm{x})-k(\mathrm{x}) * \sum_{n=1}^{N}\left(\mathrm{x}, x_{n}\right) m^{-1}(S) S(\mathrm{x})^{2}\right] * \phi^{*}=0
$$

for any $\phi \perp I$. By Theorem 2.1 we may then write

$$
k(\mathrm{x}) *\left\{\phi^{*}(\mathrm{x})-\sum_{n=1}^{N} \dot{a_{n}}\left(\mathrm{x}, x_{n}\right)\right\}=0
$$

where $a_{n}=M\left\{\phi(x)\left(\mathrm{x}, x_{n}\right)^{\bullet}\right\}$.

Since $k(x)$ is an arbitrary function in $L^{1}$, it follows that

$$
\phi(\mathbf{x})=\sum_{n=1}^{N} a_{n}\left(\mathbf{x}, x_{n}\right)
$$

(10) It should be pointed out that some of these papers contain theorems proved for arbitrary locally compact abelian groups. Since the present paper was submitted for publication, Dr. Henry Helson has kindly sent me a reprint of his paper Spectral synthesis of bounded functions, Arkiv för Matematik vol. 1 (1951) pp. 497-602. This paper contains also a generalization of Wiener's theorem (for arbitrary locally compact abelian groups). The theorem is stated there in a weaker form, but the proof would actually yield the result given here. 
almost everywhere. Cf. [6, Hypothẹse A, p. 136] and [9].

If $Z_{I}$ is discrete, then any uniformly continuous $\phi \perp I$ is almost periodic, with spectrum in $Z_{I}$ (and conversely).

Let $k_{C}(x)$ be any function in $L^{1}$ whose Fourier transform vanishes outside some compact set $C$. Then, in the same way as before,

$$
k_{C}(\mathrm{x}) * \boldsymbol{\phi}^{*}=\sum_{\imath} k_{C}\left(x_{\imath}\right) \dot{a_{\imath}}\left(\mathrm{x}, x_{\imath}\right) \quad\left(x_{\imath} \in Z_{I} \cap C\right) .
$$

Now the functions of the type $k_{C}$ are dense in $L^{1}$ [13, Theorem 2.6]. Thus for any $k \in L^{1}$ the function $k * \phi$ is almost periodic, with spectrum in $Z_{I}$. In particular, set $k(x)=m^{-1}(V) V(x)$, where $V(x)$ is the characteristic function of a neighborhood $V$ of $e$. Given $\epsilon>0$, there is a $V$ such that $\left|m^{-1}(\boldsymbol{V}) \boldsymbol{V}(\mathbf{x}) * \boldsymbol{\phi}-\boldsymbol{\phi}(\mathbf{x})\right|<\boldsymbol{\epsilon}$, for all $\mathbf{x} \in \boldsymbol{G}$, by the assumed uniform continuity of $\boldsymbol{\phi}(\mathbf{x})$. Hence $\boldsymbol{\phi}(\mathbf{x})$ itself is almost periodic, with spectrum in $Z_{I}$ (the converse follows from the approximation theorem for almost periodic functions).

For the case $G=R^{1}$ this was proved by A. Beurling [2].

If now $G=R^{p}$, let $V_{n}$ be a sequence of ( $p$-dimensional) cubes, with center at the origin and sides of length $1 / n$. Then $m^{-1}\left(V_{n}\right) V_{n}(\mathbf{x}) * \boldsymbol{\phi} \rightarrow \boldsymbol{\phi}(\mathbf{x})$ almost everywhere $(n \rightarrow \infty)$. Since $m^{-1}\left(V_{n}\right) V_{n}(\mathbf{x}) * \boldsymbol{\phi}$ is almost periodic, there are trigonometric polynomials $P_{n}(\mathrm{x})$, with frequencies in $Z_{I}$, such that $\left|m^{-1}\left(V_{n}\right) V_{n}(\mathbf{x}) * \phi-P_{n}(\mathbf{x})\right|<1 / n, n \geqq 1$. Thus $P_{n}(\mathbf{x}) \rightarrow \boldsymbol{\phi}(\mathbf{x})$ almost everywhere $(n \rightarrow \infty)$ and $\left|\boldsymbol{P}_{n}(\mathbf{x})\right|<\|\boldsymbol{\phi}\|_{\infty}+1$ for all $n$. Hence, for $G=\boldsymbol{R}^{p}$, if $Z_{I}$ is discrete, then the set of all $\phi \perp I$ consists of all those functions in $L^{\infty}$ which are limits (almost everywhere) of sequences of uniformly bounded trigonometric polynomials with frequencies in $Z_{I}$.

In the case $G=R^{1}$ this was proved by Carleman [3, p. 115], with an additional restriction on $Z_{I}$.

If $Z_{I}$ is discrete, then to any $\phi \perp I$ there corresponds (by Theorem 2.1) the "Fourier series"

$$
\boldsymbol{\phi}(\mathbf{x}) \sim \sum a_{\iota}\left(\mathrm{x}, x_{\iota}\right)
$$

where $a_{\imath}=M\left\{\boldsymbol{\phi}(\mathbf{x})\left(\mathbf{x}, x_{\imath}\right)^{\cdot}\right\}$ and the summation extends over all $x_{\iota} \in Z_{I}$. The "sum" is purely formal-no ordering of the terms, or convergence, is implied.

If all the Fourier coefficients are zero, then $\phi(x)$ vanishes almost everywhere ("uniqueness theorem"), since then $\phi \perp L^{1}$.

"Bessel's inequality" holds in the sense that

$$
\sum\left|a_{\imath}\right|^{2} \leqq \liminf _{n \rightarrow \infty} m^{-1}\left(S_{n}\right) \int S_{n}(\mathbf{x})^{2}|\boldsymbol{\phi}(\mathbf{x})|^{2} d \mathbf{x},
$$

the set of all $a_{\imath} \neq 0$ being denumerable. The proof is the same as in the classical case.

The question now arises under what conditions it is true that 


$$
\lim _{n \rightarrow \infty} m^{-1}\left(S_{n}\right) \int S_{n}(\mathbf{x})^{2}|\phi(x)|^{2} d x=\sum\left|a_{\imath}\right|^{2}
$$

("Parseval's equation").

Consider the function

$$
\boldsymbol{\alpha}(\mathbf{x})=m^{-1}(S) \int \boldsymbol{S}(\mathbf{y})^{2} \boldsymbol{\phi}(\boldsymbol{y} \mathbf{x}) \boldsymbol{\phi}^{\cdot}(y) d y,
$$

which is orthogonal to $I$. We have, for large $n$,

$$
\begin{aligned}
& M\left\{\boldsymbol{\alpha}(\mathbf{x})\left(\mathbf{x}, x_{\imath}\right)^{\cdot}\right\}=\int m^{-1}\left(S_{n}\right) \boldsymbol{S}_{n}(\mathbf{x})^{2}\left(\mathbf{x}, x_{\imath}\right)^{\bullet} d x \int m^{-1}(S) \boldsymbol{S}(\mathbf{y})^{2} \boldsymbol{\phi}(y \mathbf{x}) \boldsymbol{\phi}^{\bullet}(\mathbf{y}) d \mathbf{y} \\
& =\int m^{-1}(S) S(y)^{2} \boldsymbol{\phi}^{\bullet}(y) d y \int m^{-1}\left(S_{n}\right) S_{n}(\mathbf{x})^{2}\left(\mathbf{x}, x_{\imath}\right)^{\cdot} \boldsymbol{\phi}(y \mathbf{x}) d \mathbf{x} \\
& =\int m^{-1}(S) \boldsymbol{S}(\mathbf{y})^{2} \boldsymbol{\phi}^{\cdot}(\mathbf{y}) d \mathbf{y} \int m^{-1}\left(S_{n}\right) S_{n}\left(\mathbf{y x}^{-1}\right)^{2}\left(y \mathbf{x}^{-1}, x_{\imath}\right) \boldsymbol{\phi}(\mathbf{x}) d \mathbf{x} .
\end{aligned}
$$

But, in view of Theorem 2.1, this is just

$$
\begin{aligned}
\int m^{-1}(S) S(y)^{2} \boldsymbol{\phi}^{\cdot}(y) a_{\iota}\left(y, x_{\imath}\right) d y & \\
& =a_{\imath} m^{-1}(S) \int S\left(y^{-1}\right)^{2}\left(y^{-1}, x_{\imath}\right) \phi^{*}(y) d y \\
& =a_{\iota} \sum_{\kappa} \Delta(\iota, \kappa) \dot{a_{\kappa}} \quad\left(\Delta(\iota, \kappa)=m^{-1}(S) S * S\left(x_{\iota} x_{\kappa}{ }^{-1}\right),\right.
\end{aligned}
$$

by an argument already familiar. The last sum (over all $x_{x} \in Z_{I}$ ) has actually only a finite number of terms.

Set now $S=S_{n}$ and write correspondingly $\boldsymbol{\alpha}_{n}(\mathbf{x}), \Delta_{n}(\iota, \kappa)$. We are going to indicate some restriction on $Z_{I}$ which will guarantee the absolute convergence of

$$
\sum_{\iota, k} \Delta_{n}(\iota, \kappa) a_{\imath} \dot{a_{\kappa}} .
$$

Suppose $Z_{I}$ is such that, for some $S, x S \cap Z_{I}=x$, for all $x \in Z_{I}\left(Z_{I}\right.$ may then be called "uniformly discrete"). Then we have, for sufficiently large $n$,

$$
\alpha_{n}(e)=\sum_{\imath}\left|a_{\imath}\right|^{2},
$$

by the uniqueness theorem.

Suppose now that $Z_{I}$ is the union of a finite number $N$ of sets, each uniformly discrete. Then for sufficiently large $n$, say $n \geqq n_{0}$, the following holds: 
if one of the indices $\iota, \kappa$ is kept fixed, then $\Delta_{n}(\iota, \kappa)>0$ for at most $N$ values of the other one.

Define now the function $\mathbf{T}(\iota, \kappa)$ by

$$
\begin{array}{ll}
\mathbf{T}(\iota, k)=1 & \text { if } \Delta_{n_{0}}(\iota, k)>0, \\
\mathbf{T}(\iota, k)=0 & \text { if } \Delta_{n_{0}}(\iota, k)=0 .
\end{array}
$$

Then both $\sum_{\kappa} \mathbf{T}(\iota, \kappa)$ and $\sum_{\iota} \mathbf{T}(\iota, \kappa)$ are at most equal to $N$. Hence

$$
\begin{aligned}
\sum_{\iota, \kappa} \mathrm{T}(\iota, \kappa)\left|a_{\iota}\right|\left|a_{\kappa}\right| & \leqq \sum_{\iota, \kappa} \mathrm{T}(\iota, \kappa)\left(\left|a_{\iota}\right|^{2}+\left|a_{\kappa}\right|^{2}\right) / 2 \\
& \leqq N \sum_{\iota}\left|a_{\iota}\right|^{2} .
\end{aligned}
$$

Hence the series

$$
\sum_{\iota, \kappa} \Delta_{n}(\iota, \kappa) a_{\iota} a_{\kappa} \cdot
$$

will converge absolutely, and (some order of the terms having been fixed) uniformly with respect to $n$. By the uniqueness theorem

$$
\boldsymbol{\alpha}_{n}(\boldsymbol{e})=\sum_{\imath, \kappa} \Delta_{n}(\iota, \kappa) a_{\imath} \dot{a_{\kappa}} \cdot
$$

Thus

$$
M\left\{|\boldsymbol{\phi}|^{2}\right\}=\lim _{n \rightarrow \infty} m^{-1}\left(S_{n}\right) \int S_{n}(\mathbf{x})^{2}|\boldsymbol{\phi}(\mathbf{x})|^{2} d \mathbf{x}
$$

exists and

$$
M\left\{|\boldsymbol{\phi}|^{2}\right\}=\sum_{\iota}\left|a_{\imath}\right|^{2}
$$

Thus, if $Z_{I}$ may be decomposed into a finite number of sets each of which is uniformly discrete (p. 424), then "Parseval's equation" holds for all $\phi \perp I$. It follows that the Fourier series of $\phi$ converges "in the mean" to $\phi$.

For the case $G=R^{1}$, this means that all $\phi \perp I$ are almost periodic $B^{2}$ (Besicovitch), since in this case $M\left\{|\phi|^{2}\right\}$, as defined here, coincides with the mean used by Besicovitch, in view of a theorem of Wiener [16, Theorem 21].

\section{Appendix ( $\left.{ }^{11}\right)$}

It is possible to modify the proof of Lemma 2.1.2, avoiding Lemma 2.1.1 entirely and dropping the assumption that the dual group possess a denumerable fundamental system of neighborhoods of the identity (first axiom of countability). The modification is as follows:

In Lemma 2.1.2 it is proved that the function

(11) Added September 15, 1952. 


$$
m^{-1}(S) \mathbf{S}(\mathbf{x})^{2} * \boldsymbol{\phi},
$$

for $S$ sufficiently small, is independent of $S$, without any recourse to the first axiom of countability (it is convenient, of course, to change the notation, writing, say, $S^{\prime}, S^{\prime \prime}$ instead of $S_{n}, S_{N}$, respectively, where $\left.S^{\prime} \subset S^{\prime \prime}\right)$.

To show that the function above is also independent of $x$, take $x=e$ and set

$$
m^{-1}(S) S(e)^{2} * \boldsymbol{\phi}=M .
$$

Now the difference

$$
m^{-1}(S) S(\mathbf{x})^{2} * \boldsymbol{\phi}-M
$$

is equal to

$$
m^{-1}\left(S^{\prime}\right)\left(\mathbf{S}^{\prime}(\mathbf{x})^{2} * \boldsymbol{\phi}-m^{-1}\left(S^{\prime}\right) \mathbf{S}^{\prime}(\mathbf{e})^{2} * \boldsymbol{\phi},\right.
$$

for any $S^{\prime} \subset S$, and hence we have

$$
\begin{aligned}
\left|m^{-1}(S) S(x)^{2} * \boldsymbol{\phi}-M\right| & =m^{-1}\left(S^{\prime}\right)\left|\int\left\{S^{\prime}\left(x y^{-1}\right)^{2}-S^{\prime}\left(y^{-1}\right)^{2}\right\} \phi(y) d y\right| \\
& \leqq m^{-1}\left(S^{\prime}\right)\left\|S^{\prime}\left(x^{-1} y\right)^{2}-S^{\prime}(y)^{2}\right\|_{1}\|\phi\|_{\infty} \\
& \leqq \epsilon\|\phi\|_{\infty},
\end{aligned}
$$

by property (ii), p. 404 , if $S^{\prime}$ is small enough. Thus Lemma 2.1 .2 is established by a method which is more general as well as simpler.

The only other place where the first axiom of countability is used is on p. 421 where it is proved that in the equation

$$
m^{-1}(S) S(\mathbf{x})^{2} * k * \phi^{*}=M \cdot\left\{\phi_{1}\right\}
$$

the constant $M \cdot\left\{\phi_{1}\right\}$ is zero. Keeping $S$ fixed and setting

$$
m^{-1}(S) S(\mathbf{x})^{2} * \boldsymbol{\phi}=\psi(\mathbf{x}),
$$

we have

$$
k * \psi^{*}=M \cdot\left\{\phi_{1}\right\}
$$

and also

$$
\int k(\mathrm{x}) d \mathrm{x}=0 .
$$

Now in Lemma 1.1.2 (part I) it is proved, without any countability restriction, that the last two equations imply

$$
M \cdot\left\{\phi_{1}\right\}=0 .
$$

It results that the proof of the extension of the theorem of MandelbrojtAgmon (Theorem 2.2) is valid for locally compact abelian groups in general. 


\section{BibliogRAPHY}

1. W. Ambrose, Direct sum theorem for Haar measures, Trans. Amer. Math. Soc. vol. 61 (1947) pp. 122-127.

2. A. Beurling, Sur une classe de fonctions presque-périodiques, C.R. Acad. Sci. Paris vol. 225 (1947) pp. 326-328.

3. T. Carleman, L'intégrale de Fourier et questions qui s'y rattachent, Uppsala, 1944.

4. H. Cartan and R. Godement, Théorie de la dualité et analyse harmonique dans les groupes abéliens localement compacts, Ann. Ecole Norm. vol. 64 (1947) pp. 79-99.

5. V. Ditkin, On the structure of ideals in certain normed rings, cf. Mathematical Reviews vol. 1 (1940) p. 336.

6. R. Godement, Théorèmes taubêriens et théorie spectrale, Ann. École Norm. vol. 64 (1947) pp. $119-138$.

7. P. R. Halmos, Measure theory, New York, 1950.

8. E. Hille, Functional analysis and semi-groups, Amer. Math. Soc. Colloquium Publications, vol. 31, 1948.

9. I. Kaplansky, Primary ideals in group algebras, Proc. Nat. Acad. Sci. U.S.A. vol. 35 (1949) pp. 133-136.

10. G. W. Mackey, Functions on locally compact groups, Bull. Amer. Math. Soc. vol. 56 (1950) pp. 385-412.

11. S. Mandelbrojt and $\mathrm{S}$. Agmon, Une généralisation du thêorème tauberien de Wiener, Acta Szeged vol. 12 B (1950) pp. 167-176.

12. L. Schwartz, Sur une propriêté de synthèse spectrale dans les groupes non compacts, C.R. Acad. Sci. Paris vol. 227 (1948) pp. 424-426.

13. I. E. Segal, The group algebra of a locally compact group, Trans. Amer. Math. Soc. vol. 61 (1947) pp. 69-105.

14. A. Weil, L'intégration dans les groupes topologiques et ses applications, Paris, 1940.

15. N. Wiener, Tauberian theorems, Ann. of Math. vol. 33 (1932) pp. 1-100.

16. - The Fourier integral and certain of its applications, Cambridge University Press, 1933.

RICE INSTITUTE, Houston, Tex.

UNIVERSITY OF OREGON, EUGENE, OREGON 